\title{
ATYPICAL CLINICAL VARIANTS IN NEW WORLD CUTANEOUS LEISHMANIASIS: DISSEMINATED, ERYSIPELOID, AND RECIDIVA CUTIS DUE TO LEISHMANIA (V.) PANAMENSIS
}

\author{
MANUEL CALVOPINA** EDUARDO A. GOMEZ, HIROSHI UEZATO, HIROTOMO KATO, SHIGEO NONAKA, AND \\ YOSHIHISA HASHIGUCHI \\ Department of Parasitology, Kochi Medical School, Kochi University, Kochi, Japan; Departamento de Medicina Tropical, Facultad \\ de Medicina, Universidad Catolica Santiago de Guayaquil, Ecuador; Department of Dermatology, Faculty of Medicine, University of \\ the Ryukyus, Okinawa, Japan; Department of Veterinary Hygiene, Faculty of Agriculture, Yamaguchi University, Yamaguchi, Japan
}

\begin{abstract}
In recent times, there has been an increase in the number of reports for new and rare variants of cutaneous leishmaniasis (CL). Here, we describe three unusual clinical forms of CL identified in Ecuadorian children. A total of 131 patients with CL were diagnosed over a 2-year period of active search. In 3 (2.29\%), the lesions were very unusual; these included erysipeloid, recidiva cutis (LRC), and disseminated leishmaniasis (DL). The erysipeloid case is characterized by erythematous and indurated plaque seen on the face of a 5-year-old boy; the LRC one is differentiated by slowly progressing red-brown papules around large scars of healed sores in a 6-year-old girl, and the DL case is characterized by dozens of cutaneous ulcers distributed in the whole body of a 1-year-old girl. Leishmania parasites were isolated by lesion aspirate and analyzed by the technique multilocus enzyme electrophoresis (MLEE). All three isolates were identified as Leishmania (Viannia) panamensis. These distinct clinical variants rarely have been reported previously in the American cutaneous leishmaniasis, and for the first time $L$. (V.) panamensis was identified as the etiologic agent. Our cases extend the spectrum of clinical presentations in New World leishmaniasis.
\end{abstract}

\section{INTRODUCTION}

New World tegumentary leishmaniasis is best known for the destructive, mutilating lesions of mucocutaneous leishmaniasis (MCL) or "espundia." However, cutaneous leishmaniasis (CL) includes a variety of clinical forms. The most common clinical form is the "classical" ulcer, which starts as a nodule, becomes an ulcer with an indurated raised outer border and sharply incised central crater, and then usually heals over a period of months. But, some lesions do not ulcerate and heal but persist as spreading nodules as in diffuse cutaneous leishmaniasis (DCL) and in the atypical CL of Central American countries. ${ }^{1}$ Another clinical variant, "Uta," is seen in the highlands of Peru and Ecuador and is characterized by small, usually single crusted papules. ${ }^{2,3}$ Verrucous, Chiclero ulcer, and sporotrichoid lesions (pian-bois) are also described.

Disseminated cutaneous leishmaniasis (DL) and leishmaniasis recidiva cutis (LRC) are rare variants. DL is characterized by the appearance of multiple pleomorphic lesions in $>2$ noncontiguous areas of the body and has been reported exclusively from Brazil ${ }^{4}$ and a solitary case from French Guiana and Ecuador. ${ }^{5,6}$ LRC is characterized by red-brown papules that appear in or around the scar of a healed sore. ${ }^{7}$ The erysipeloid form, which is characterized by erythematous, indurated swollen plaques without ulceration, has not been yet reported in the New World. Other unusual variants are reported, but in the Old World, such as paronychial, chancriform, annular, palmo-plantar, erysipeloid, zosteriform, Whitlow, and sporotrichoid..$^{8-10}$

Determinants of the clinical presentations of disease are poorly understood but include factors related to the Leishmania species, the host's immune response, and, possibly, the saliva of the sandfly vector. ${ }^{11}$ At least 16 different Leishmania spp. have been identified as causative agents in the human New World leishmaniasis falling into both subgenera Leish-

\footnotetext{
* Address correspondence to Manuel Calvopina, Department of Parasitology, Kochi Medical School, Kochi University, Kochi 7838505, Japan. E-mail: mcalvopina@hotmail.com
}

mania and Viannia. ${ }^{12}$ It is generally accepted that certain species predominantly cause major clinical forms; for example, $L$. (L.) chagasi for the visceral form and $L$. (V.) braziliensis for MCL. However, many of the parasites are capable of producing in the human host a spectrum of disease rather than a single clinical form, thus L. (L.) amazonensis is associated with CL, MCL, post Kala-azar dermal leishmaniasis, and the visceral form. ${ }^{13}$

The usual clinical presentations of leishmaniasis are easily diagnosed by clinicians in endemic regions, but the unusual forms may give rise to difficulties in diagnosis and appropriate treatment. Here, we report three children with atypical variants of CL acquired in Ecuador, in whom the parasites were identified as $L$. (V.) panamensis by multilocus enzyme electrophoresis (MLEE).

\section{MATERIALS AND METHODS}

An active search for leishmaniasis was undertaken in the three natural regions of Ecuador (Pacific coastal, Andean, and Amazonian) in the period 2001-2003. Diagnosis of leishmaniasis was confirmed by the demonstration of amastigotes in slit smears and/or the presence of flagellated promastigotes in the USMARU medium. ${ }^{14}$ Parasite strain characterization was done for 11 different enzyme systems using cellulose acetate electrophoresis enzyme technique. Enzymes include 4 oxido-reductases, 2 isomerases, 4 transferases, and 1 hydrolase. Technical conditions for preparation of samples, electrophoresis, and staining procedures were followed as described by Evans. ${ }^{14}$ For profile comparisons, WHO reference strains included in this study were as follows: L. amazonensis (MHOM/BR/73/M2269), L. mexicana (MNYC/BZ/62/M379), L. braziliensis (MHOM/BR/75/M2904), L. panamensis (MHOM/PA/71/LS94), and L. guyanensis (MHOM/BR/75/ M4147). We also included L. chagasi (MHOM/BR/74/M2682) because is reported to be the causative agent of nodular atypical CL in Central American countries. The genetic relationship among the stocks were established according Sneath and Sokal. ${ }^{15}$ 


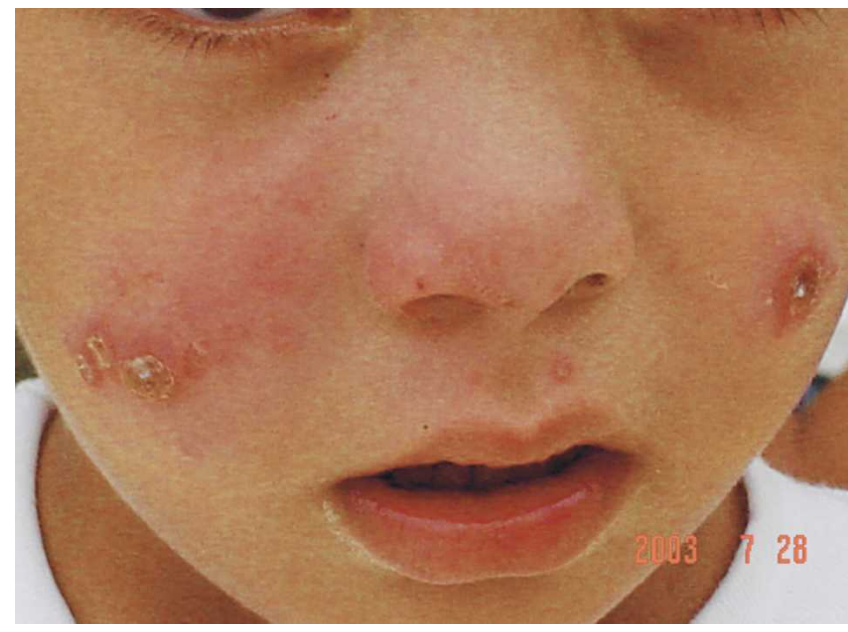

FIGURE 1. Clinical image of patient 1 , erythematous and indurated plaque in both cheeks joined by a large infiltrate thorough upper lip and nose. A punch biopsy had been performed 2 weeks ago at the center of the plaques.
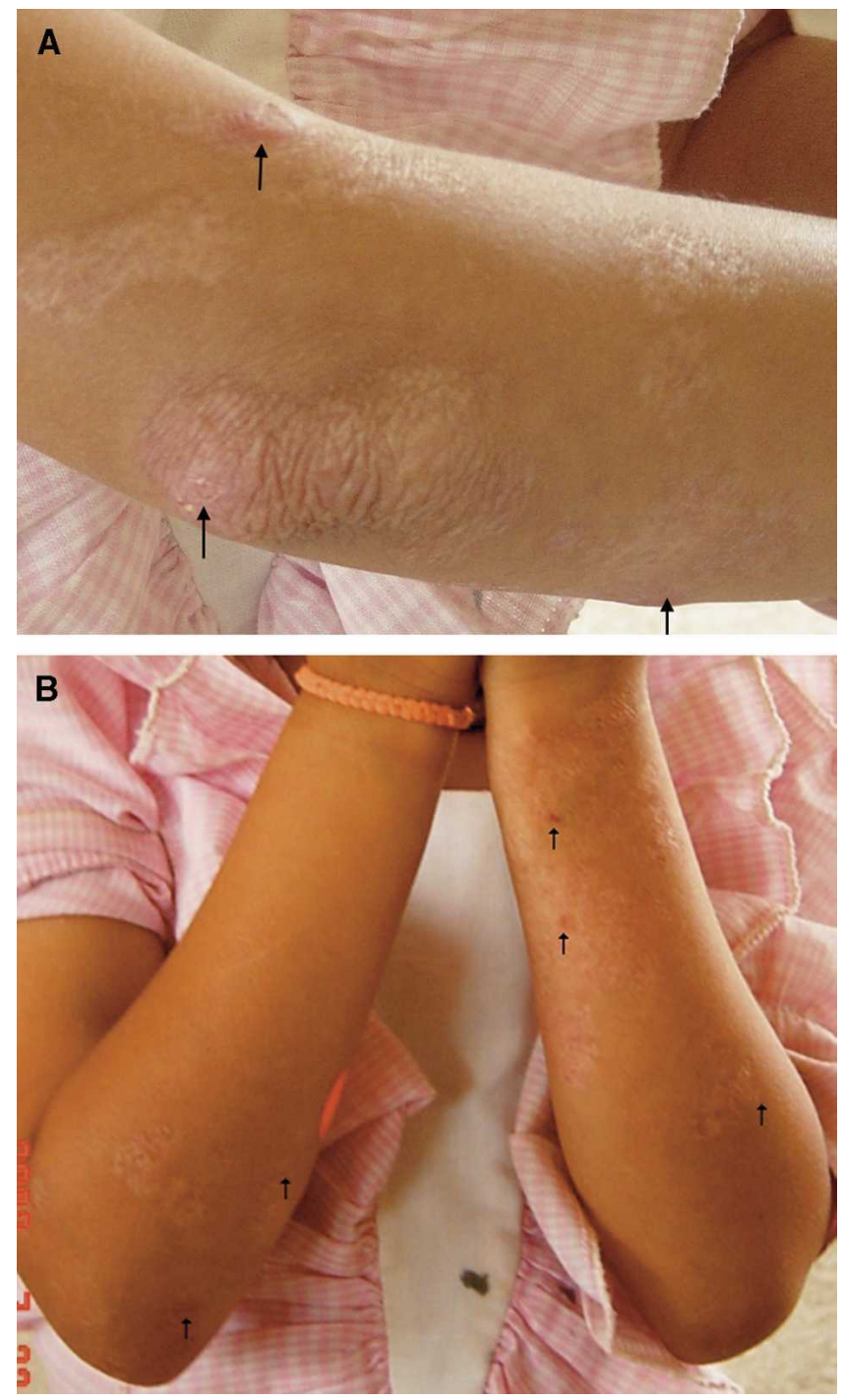

Figure 2. A and B. Photographs of patient 2 showing typical recidivans cutaneous lesion, with several small red-brown papules (arrows) at the edges of extensive scars where Leishmania parasites were isolated.
All patients received treatment consisting of parenteral injections of meglumine antimoniate (Glucantime; $20 \mathrm{mg} \mathrm{Sb}$ $\mathrm{kg}^{-1}$ day $^{-1}$ for 15 days) as is recommended by the Ecuadorian Ministry of Public Health. The study adhered to the ethical rules of the Central University of Ecuador in accordance with Ecuadorian government law.

\section{RESULTS}

A total of 131 patients were diagnosed to have CL. Three of them had atypical disease and are described in this report. All three came from leishmaniasis-endemic areas of subtropical Ecuador (humid subtropical forest) at 800-1000 m elevation. Results of epidemiologic, clinical features, and treatment of the rest of the patients and characterization of their parasites are reported elsewhere.

Patient 1. A 4-year-old boy presented with a large painless erythematous lesion on the face (Figure 1). It had evolved from an initial papule in the left cheeck first noticed 5 months earlier and which gradually spread. The lesion was treated

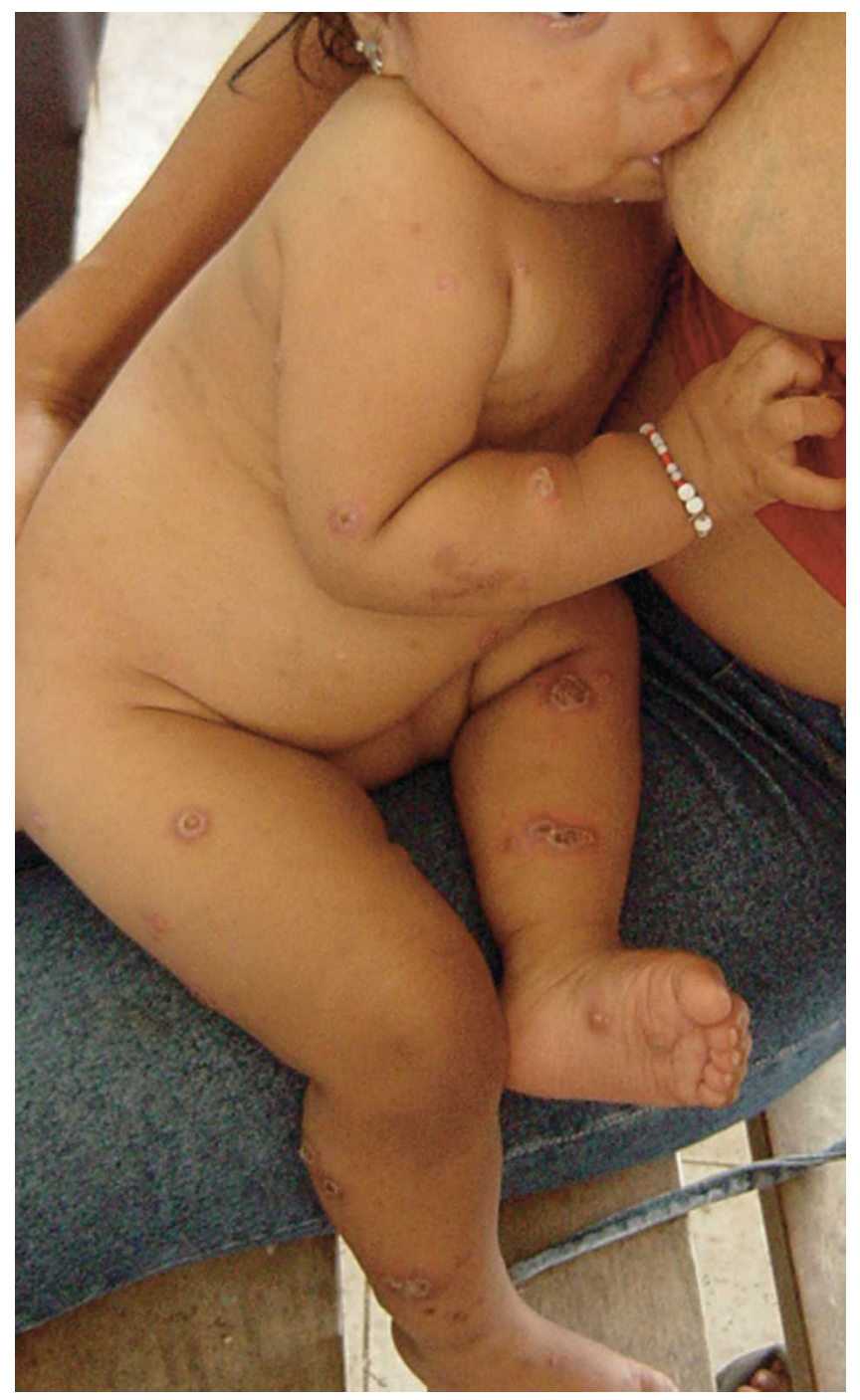

FIGURE 3. Ulcerative lesions disseminated during 3 months on the head, trunk, legs, and arms in our 1-year-old patient of disseminated cutaneous leishmaniasis. Note cutaneous lesions in the sole. 
with topical steroids, followed by topical antibiotics and systemic penicillin, but without improvement. On examination, we found a well-nourished boy, without fever, with a painless, erythematous and indurated plaque on cheeks, nose, and upper lip, but without lesions on the mucous membranes.

Patient 2. A 6-year-old girl presented with a 3-year history of seven red-brown papules slowly expanding at the periphery of healed sores on her right and left upper limbs involving arms, elbows, and forearms. Physical examination of her right arm showed three extensive atrophic scars of healed sores, with four small, red-brown papules at their periphery, and on her left forearm three small red papules in the periphery of extensive scars. The scars were atrophic, ill-defined, depressed, whitish and pink in color and cosmetically disfiguring (Figure 2). These cutaneous changes were suspected to be cutaneous tuberculosis by the local physician. Detailed questioning revealed that scars represented previous ulcers that had healed after a year of evolution, without any treatment, and that after a further year the disease reactivated in the border of healed lesions and continued spreading at the borders.

Patient 3. A 1-year-old girl presented with dozens of cutaneous ulcers distributed in her head, trunk, arms, and legs (Figure 3). Past medical history revealed that approximately 4 months earlier, her mother observed a solitary ulcer on the child's left thigh. At the same time, the mother and a brother had similar but localized ulcers. After a week, the patient developed multiple new skin lesions disseminated over different regions of the body, which persisted. The child suffered from chronic malnutrition. The local physician diagnosed chicken pox at first, but later a generalized impetigo, and prescribed topical and parenteral antibiotics, to which she showed no response. On physical examination, a total of 69 lesions, either between crusted papules and ulcers, were counted on all parts of the body even on the palms and soles, but mucous membranes were not affected.

Diagnosis and identification of Leishmania isolates. Microscopy showed intracellular Leishmania, and culture yielded promastigotes. The three isolated stocks were identified as $L$. (V.) panamensis by comparing their enzyme profiles for 11 enzyme systems with the profiles of those of selected WHO reference strains. The most useful system to differentiate between subgenera and among Viannia species was the phosphogluconate dehydrogenase (Figure 4), but differences were also found in the glucose-6-phosphate dehydrogenase and nucleoside hydrolase. The UPGMA dendrogram built from the Jaccard's distance matrix drew the same cluster (similar isoenzyme profile) between our three stocks and $L$. (V.) panamensis reference stock (data not shown). L. chagasi migrated far from our samples. Treatment resulted in clinical cure in all three patients.

\section{DISCUSSION}

Because of the rarity of the atypical clinical forms of cutaneous leishmaniasis reported here, even physicians working in such an endemic area misdiagnosed them as chicken pox, erysipelas, and cutaneous tuberculosis and therefore patients were treated inappropriately. The rarity of these variants in the current study $(2.29 \%)$ is corroborated by other surveys carried out in the country and in other endemic regions in the New and Old Worlds. ${ }^{1,4,9,10,16}$ Lesions at unusual sites are

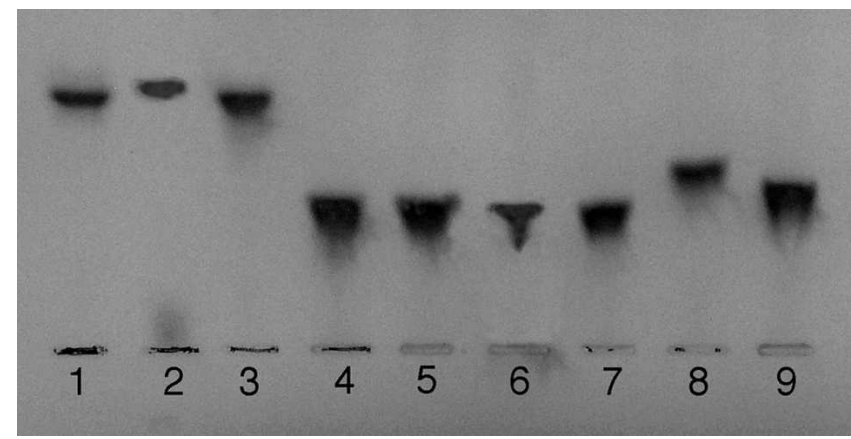

FIGURE 4. Comparision of the isoenzyme pattern for 6-phosphogluconate deshydrogenase enzyme system of reference strains (WHO recommended) with our three isolates. Lane 1, L. mexicana. Lane 2, L. amazonensis. Lane 3, L. chagasi. Lanes 4-6, patients 1-3, respectively. Lane 7, L. panamensis. Lane 8, L. guyanensis. Lane 9, L. braziliensis.

also rarely reported, but several lesions on palms and soles occurred in our 1-year-old patient.

The differential diagnosis of "classical" ulcers of CL includes infected insect bites, traumatic ulcers, fungal, mycobacterial, and spirochete infections, and squamous cell carcinoma. ${ }^{17}$ Disseminated leishmaniasis (DL) should be differentiated from small pox, paracoccidioidomycosis, and staphylococcal infection ${ }^{6}$ and as in our case with chicken pox and impetigo; in addition, lesions in Brazilian patients resembled leprosy and acne. ${ }^{4}$ The erysipeloid form on the face should be distinguished from systemic lupus erythematosus, cellulitis and erysipelas, rosacea with lymphoedema, and sarcoidosis. LRC is commonly misdiagnosed as lupus vulgaris, but blastomycosis, sarcoidosis, and tinea incognita must be also considered. ${ }^{18}$

Interestingly and for the first time in the clinical spectrum of American tegumentary leishmaniasis, L. (V.) panamensis was identified in all three distinct variants of CL reported here. Previously, L. (V.) braziliensis in Brazilian patients and $L$. $(V$.$) guyanensis in a French Guiana patient were identified$ in $\mathrm{DL}^{4,5}$ and $L$. (L.) amazonensis and $L$. (V.) braziliensis in LRC forms. ${ }^{19}$

$\mathrm{WHO}^{20}$ stated that $L$. (V.) panamensis cause lesions that are normally ulcers. However, the current findings and other reports incriminate this species as the etiological agent for other clinical forms such as Chiclero ulcers and even for mucosal and for MCL. ${ }^{21}$ As the clinical pattern of leishmaniasis is determined by the interaction between the host immune response and the strain of the parasite implicated, ${ }^{22}$ the fact showed in this report that the same parasite cause several clinical forms suggests that the host immune response is critical in determining the outcome of $L$. $(V$.) panamensis infection. Indeed, atypical clinical features and locations of Leishmania are common in patients co-infected with HIV. ${ }^{23,24}$

Estimating the importance and complexity of clinical features of leishmaniasis and skin diseases in the Tropics, it is worth reporting unusual and rare clinical forms and localizations of such diseases. Physicians and researchers need to appreciate that the local identified species of Leishmania are capable of causing atypical diseases, and familiarity with the different clinical presentation of leishmaniasis may facilitate the diagnosis, and therefore all early treatment may improve outcome. 
Received December 23, 2004. Accepted for publication April 6, 2005.

Acknowledgments: The authors acknowledge Professor Anthony Bryceson for his helpful comments on the manuscript and Hideo Kumazawa for helping with the photographic illustrations.

Authors' addresses: Manuel Calvopina and Yoshihisa Hashiguchi, Department of Parasitology, Kochi Medical School, Kochi University, Nankoku, Kochi 785-8505, Japan. Hiroshi Uezato and Shigeo Nonaka, Department of Dermatology, Faculty of Medicine, University of the Ryukyus, Nishihara, Okinawa 903-0215, Japan. Eduardo A. Gomez, Departmento de Medicina Tropical, Facultad de Medicina, Universidad Catolica Santiago de Guayaquil, Ecuador. Hirotama Kato, Department of Veterinary Hygiene, Faculty of Agriculture, Yamaguchi University, 1677-1 Yoshida, Yamaguchi 7538515, Japan.

Reprint requests: Manuel Calvopina, Department of Parasitology, Kochi Medical School, Kochi University, Kochi 783-8505, Japan, Telephone: +81 088880 2617, Fax: +81 088880 2415, E-mail: mcalvopina@hotmail.com.

\section{REFERENCES}

1. Belli A, Garcia D, Palacios X, Rodriguez B, Valle S, Videa E, Tinoco E, Marin F, Harris E, 1999. Widespread atypical cutaneous leishmaniasis caused by Leishmania (L.) chagasi in Nicaragua. Am J Trop Med Hyg 61: 380-385.

2. Lucas CM, Franke ED, Cachay MI, Tejada A, Cruz ME, Kreutzer RD, Barker DC, McCann SHE, Watts DM, 1998. Geographical distribution and clinical description of leishmaniasis cases in Peru. Am J Trop Med Hyg 59: 312-317.

3. Hashiguchi Y, Gomez EA, de Coronel VV, Mimori T, Kawabata M, Furuya M, Nonaka S, Takaoka H, Alexander JB, Quizhpe AM, Grimaldi G Jr, Kreutzer RD, Tesh RB, 1991. Andean leishmaniasis in Ecuador caused by infection with Leishmania mexicana and L. major-like parasites. Am J Trop Med Hyg 44: 202-217.

4. Turetz ML, Machado PR, Ko AI, Alves F, Bittencourt A, Almeida RP, Mobashery N, Johnson WD Jr, Carvalho EM, 2002. Disseminated leishmaniasis: A new and emerging form of leishmaniasis observed in Northeastern Brazil. J Infect Dis 186: $1829-1834$.

5. Couppie P, Clyti E, Sainte-Marie D, Dedet JP, Carme B, Pradinaud R, 2004. Disseminated cutaneous leishmaniasis due to Leishmania guyanensis: Case of a patient with 425 lesions. Am J Trop Med Hyg 71: 558-560.

6. Lazo R, Hashiguchi Y, 1994. Generalized cutaneous leishmaniasis: a parasitologically confirmed case in Ecuador. Hashiguchi Y, ed. Studies on New World Leishmaniasis and Its Transmission, with Particular Reference to Ecuador. Research Report Series 4. Japan: Kyowa Printing, 93-98.

7. Paes-Oliveira M, 1997. Leishmaniasis recidiva cutis. Ann Bras Dermatol 52: 353-359.

8. Karincaoglu Y, Esrefoglu M, Ozcan H, 2004. Atypical clinical form of cutaneous leishmaniasis: erysipeloid form. Int $J$ Dermatol 43: 827-829.

9. Iftikhar N, Bari I, Ejaz A, 2003. Rare variants of cutaneous leishmaniasis: Whitlow, paronychia and sporotrichoid. Int J Dermatol 42: 807-809.

10. Raja KM, Khan AA, Hameed A, Rahman S, 1998. Unusual clinical variants of cutaneous leishmaniasis in Pakistan. Br J Dermatol 139: 111-113.

11. Grimaldi G Jr, Tesh RB, 1993. Leishmaniasis of the New World: current concepts and implications for future research. Clin Microbiol Rev 6: $230-250$.

12. Shaw JJ, 2002. The ecology of leishmaniasis and the diversity of leishmanial species in Central and South America. Farrel JP, ed. World Class Parasites: Leishmania. Volume 4. New York: Kluwer Academic Publishers, 11-31.

13. Barral A, Pedral-Sampaio D, Grimaldi G Jr, Momen H, McMahon-Pratt D, Ribeiro de Jesus A, Almeida R, Badaro R, Barral-Netto M, Carvalho EM, Johnson WD Jr, 1991. Leishmaniasis in Bahia, Brazil: evidence that Leishmania amazonensis produces a wide spectrum of clinical disease. Am J Trop Med Hyg 44: 536-546.

14. Evans D, 1989. Handbook on Isolation, Characterization and cryopreservation of Leishmania. WHO/TDR, 12-11 Geneva, Switzerland, 1-45.

15. Sneath PHA, Sokal RR, 1973. Sneath PHA, Sokal RR, eds. Numerical Taxonomy. San Francisco: W.H. Freeman, 1-487.

16. Armijos RX, Weigel MM, Izurieta R, Racines J, Zurita C, Herrera W, Vega M, 1997. The epidemiology of cutaneous leishmaniasis in subtropical Ecuador. Trop Med Int Health 2: 140152.

17. Hepburn NC, 2000. Cutaneous leishmaniasis. Clin Exp Dermatol 25: $367-370$.

18. Griffiths WAD, 1987. Old World cutaneous leishmaniasis. Peters $\mathrm{W}$, Killick-Kendrick R, eds. The Leishmaniases in Biology and Medicine. Volume 2. London: Academic Press, 617-636.

19. Bittencourt AL, Costa JML, Carvalho EM, Barral A, 1993. Leishmaniasis recidiva cutis in American cutaneous leishmaniasis. Int J Dermatol 32: 802-805.

20. World Health Organization, 1984. The Leishmaniases. Technical Report Series 701. Geneva: WHO, 1-140.

21. Osorio LE, Castillo CM, Ochoa MT, 1998. Mucosal leishmaniasis due to Leishmania (Viannia) panamensis in Colombia: clinical characteristics. Am J Trop Med Hyg 59: 49-52.

22. Bryceson ADM, 1986. Clinical variations associated with taxa of Leishmania. Col Int CNRS/Interm 1948 Montpelier, France: IMEEE, 221-228.

23. Rosenthal E, Marty P, del Giudice P, Pradier C, Ceppi C, Gastaut JA, Fichoux Y, Cassuto JP, 2000. HIV and Leishmania coinfection: a review of 91 cases with focus on atypical locations of Leishmania. Clin Infect Dis 31: 1093-1095.

24. Couppie P, Clyti E, Sobesky M, Bissuel F, Del Giudice P, SainteMarie D, Dedet JP, Carme B, Pradinaud R, 2004. Comparative study of cutaneous leishmaniasis in human immunodeficiency virus (HIV)-infected patients and non-HIV-infected patients in French Guiana. Br J Dermatol 151: 1165-1171. 